\title{
A statistical evaluation of multiple regression models for contact dynamics in rail vehicles using roller rig data
}

\author{
SayedMohammad Hosseini ${ }^{\mathrm{a}^{*}}$, Ahmad Radmehr ${ }^{\mathrm{a}}$, Arash Hosseinian Ahangarnejad ${ }^{\mathrm{a}}$, Robert B. \\ Gramacy $^{\mathrm{b}}$, and Mehdi Ahmadian ${ }^{\mathrm{a}}$ \\ ${ }^{a}$ Center for Vehicle Systems and Safety (CVeSS), Virginia Polytechnique and State University, \\ Blacksburg, VA, USA, 24061 \\ ${ }^{b}$ Department of Statistics, Virginia Polytechnique and State University, Blacksburg, VA, USA, 24061
}

A statistical analysis of a large amount of data from experiments conducted on the Virginia Tech-Federal Railroad Administration (VT-FRA) roller rig under various field-emulated conditions is performed to develop multiple regression models for longitudinal and lateral tractions. The experiment-based models are intended to be an alternative to the classical wheel-rail contact models that have been available for decades. The VT-FRA roller rig data is used to develop parametric regression models that efficiently capture the relationship between traction and the combined effects of the influential variables. Single regression models for representing the individual effect of wheel load, creepage, and angle of attack on longitudinal and lateral traction were investigated by the authors in an earlier study. This study extends single regression models to multiple regression models and assesses the interaction among the variables using model selection approaches. The multiple regression models are then compared with CONTACT, a well-known modeling tool for contact dynamics, in terms of prediction accuracy. The predictions made by both CONTACT and multiple regression models for longitudinal and lateral tractions are in close agreement with the measured data on the VT-FRA roller rig. The multiple regression model, however, offers an algebraic expression that can be solved far more efficiently than a simulation run in CONTACT for a new dynamic condition. The results of the study further indicate that the established multiple regression models are an effective means for studying the effect of multiple parameters such as wheel load, creepage, and angle of attack on longitudinal and lateral tractions. Such data-driven parametric models provide an essential analysis and engineering tool in contact dynamics, just as they have in many other areas of science and engineering.

\footnotetext{
${ }^{*}$ Corresponding author. Email: mohammadhosseini@vt.edu
} 
Keywords: Statistical modeling, wheel-rail contact, roller rig, experimental data, longitudinal traction, lateral traction, creepage, angle of attack, wheel load, parametric regression

\section{Introduction}

The angle of attack (AoA), percent-creepage, and wheel load are considered to be the most important variables influencing wheel-rail contact forces, in addition to the physical attributes of the contacting surfaces such as finish, coefficient of friction, etc. There are well-known models that have evolved from the Hertzian contact over the years [1]. Various studies have investigated the nature of traction and traction forces at the wheel-rail contact based on these models. As an example, Beak et al. [2] investigated the transient traction characteristics between two rollers on a test device under dry and lubricated conditions. Spiryagin et al. modeled creep force for rail traction vehicles by modifying Kalker's algorithm [3]. Wheel-rail creep forces and their influence on derailment were studied through simulations by Santamaria et al. [4]. Similarly, Jin et al. [5] have studied how the elastic deformations of wheelset and track affect the creep forces in rolling contact. The influence of AoA and its role in derailment are investigated in [6-8]. The common attribute of most of these studies is that they are either theoretical or use experimental data to quantify the parameters in standard theories.

In contrast, Alonso et al. took an experimental approach toward characterizing the wheelrail contact dynamics and calculating contact forces by building a test bench [9]. Their approach, as well as similar efforts by others that have adopted rolling test rigs of various sizes and constructions, are intended to complement the theoretical studies by generating test data under controlled conditions that can be used to improve rail vehicle performance [10]. Such test rigs have also provided the opportunity to generate a vast amount of data that requires careful processing to extract the information of interest or provide additional learning to the contact 
mechanics theories. The data processing would often require techniques that are adaptable to uncertainties or stochastic elements that inevitably would exist in the data. Machine learning techniques and advanced predictive models have often been suggested as suitable methods for analyzing complex datasets. Their use in railroad engineering, however, has been limited even though they have been widely adopted in other fields. Among the few application of advanced data analytics approaches in rail engineering, one can point to the study by Nachtigall, et al. in the 1980s and 1990s for operational scheduling [11]. Parkinson and Iwnicki [12] also used machine learning techniques to predict wheel and rail forces toward optimizing railcar suspensions [13] in the late-1990s and early-2000s. There has been increased attention to these techniques for rail applications in recent years, although it continues to lag behind other fields in both breadth and complexity. In 2018, Shebani et al. [14] developed an artificial neural network to predict wheel-rail wear. The research showed that the neural network can be efficiently employed for this purpose. This new line of research takes an interdisciplinary approach toward solving core engineering problems aimed at maximizing efficiency and improving prediction accuracy by using advanced machine learning and deep learning techniques. For contact mechanics, however, there is a gap between the theoretical and numerical methods and the more flexible machine/deep learning approaches that are mostly focused on out-of-sample performance and accuracy of predictions. This gap could be efficiently bridged by statistically modeling the wheel-rail contact using experimental data, as intended in this study.

The experimental data generated on the Virginia Tech-Federal Railroad Administration (VT-FRA) Roller Rig is used to carry out the analysis in this study. The VT-FRA Roller Rig is an example of high precision scaled rigs that provide the means for a wider range of tests in a consistent, repeatable manner [15]. The rig consists of a $1 / 4^{\text {th }}$ scale AAR-1B railcar wheel and a 
136RE-profiled 44-inch roller that are controlled independently through two DC motors. The roller-to-wheel diameter is approximately 5:1 to reduce the contact patch distortion [15]. The two DC motors allow precisely controlling the wheel and roller rotational velocity to within 0.1 RPM, hence providing extremely precise creepage control [10]. The VT-FRA roller rig also provides precise control of the wheel load, angle of attack (AoA), lateral displacement, and cant angle. The range of variables is such that the rig can be used for a wide range of studies under precise and highly controlled conditions [16]. Wheel-rail contact patch geometry has been investigated using the measurements on the roller rig by Radmehr et al. [17].

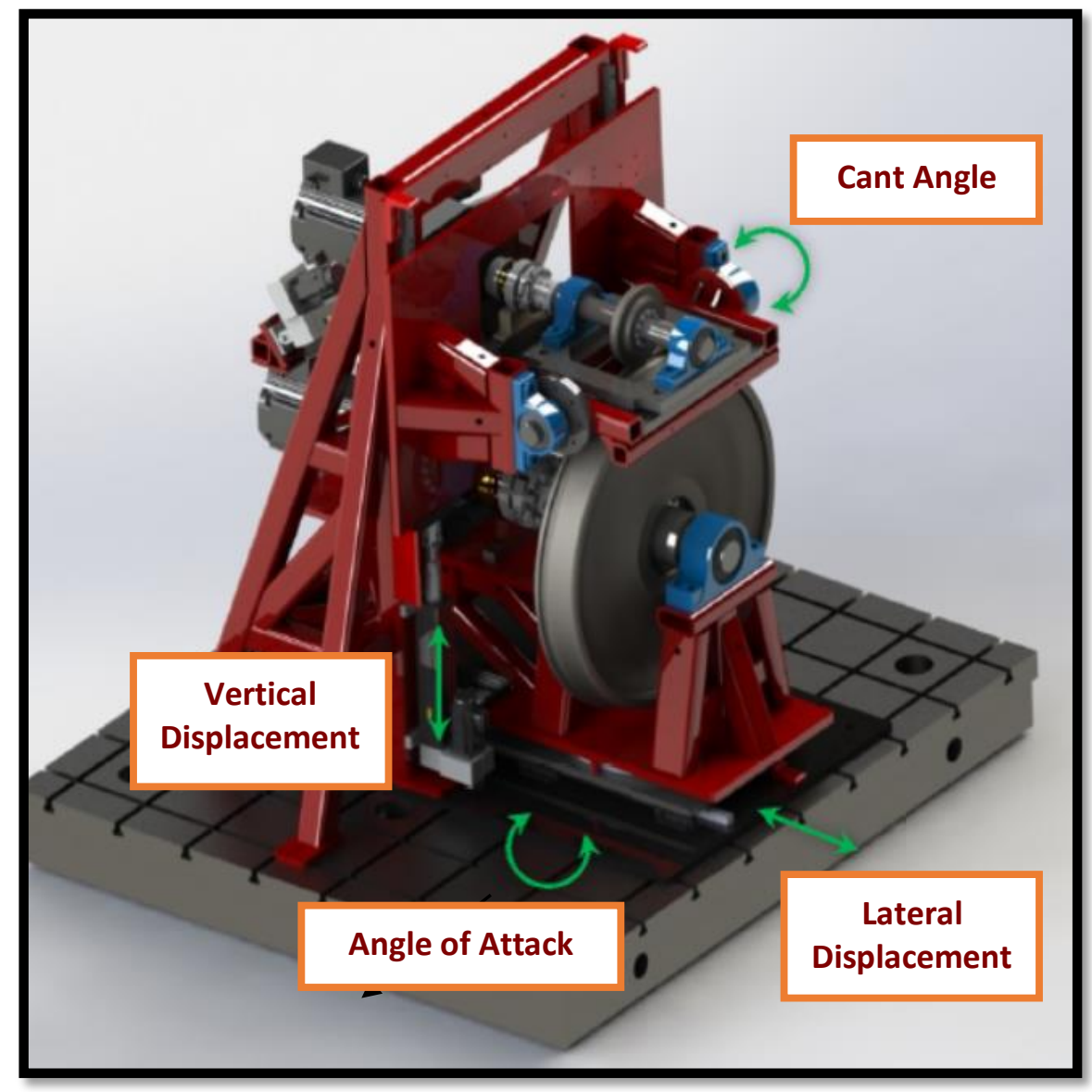

Figure 1: VT-FRA Roller Rig with the adjustable degrees of freedom 


\section{Statistical methods}

Various statistical methods are employed in this analysis including the classical linear regression model and underlying assumptions, Least Absolute Shrinkage and Selection Operator (LASSO), best subset selection, and stepwise model selection via Bayes Information Criteria (BIC). These approaches and the mathematics behind them are carefully discussed in [18].

\section{Data collection \& data sets}

A total of five experiments are carried out at different settings to study the main effects of wheel load, AoA, and creepage as well as their combined effects on traction forces. In four of the experiments, one variable is changed while others are kept constant to evaluate the effect of each one individually. The data collected in these four experiments were employed to develop single regression models to describe the effects of wheel load, AoA, and creepage on traction in an earlier study by the authors [19]. The single regression models and the corresponding data sets are discussed in detail in [18] and are not included here for brevity.

Beyond changing the variables one at a time, the fifth experiment was designed such that all variables are changed within a predetermined range, simultaneously. This experiment was targeted toward assessing the combined effect of the variables on traction forces. Although all experiments started with a cleaned and redressed wheel, the data collection started after the formation of the natural third-body layer during the first few minutes of testing [20]. This was to eliminate the effect of natural third-body layer accumulation that contributes to rising traction during the first few minutes of the tests, while the running surfaces reach their steady-state traction condition. 
The data set shown in Figure 2 measures traction forces while AoA, creepage, and wheel load are changed within the ranges shown in Table 1 simultaneously. The Simultaneous changing of the variables accommodates the condition necessary for multiple regression models, beyond the simple superposition of individual variables. The AoA and creepage vary continuously while the wheel load varies in increments specified in Table 1 . Rows $1-3$ in Figure 2 show longitudinal and lateral tractions versus AoA, creepage, and wheel load, respectively. The data is used to develop multiple regression models that relate longitudinal and lateral tractions to the three variables in the tests. $70 \%$ of the data set is randomly selected as the training set and the remaining $30 \%$ as the testing set. The training set is used to train multiple regression models and the testing set is used to evaluate the out-of-sample performance of the trained models.

Table 1: Range of independent variables in the experiment

\begin{tabular}{cc}
\hline Variable & Range / Increments \\
\hline AoA & -1 to $1(\mathrm{deg})$ \\
Creepage & 0 to $2(\%)$ \\
Wheel Load & $1500,2700,4500,9800(\mathrm{~N})$ \\
\hline
\end{tabular}



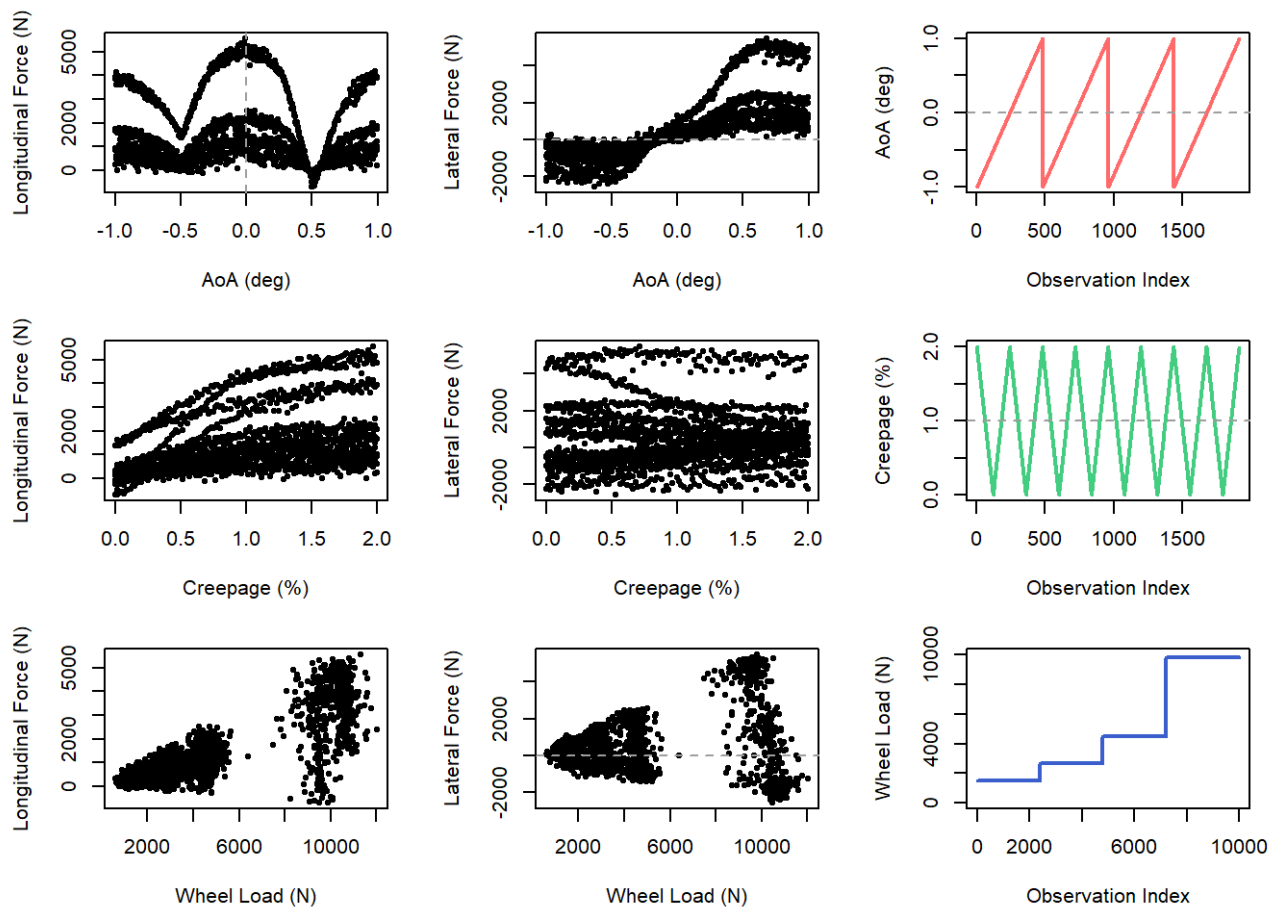

Figure 2: Measurements of longitudinal and lateral forces while changing AoA and creepage continuously at various wheel load increments

Please note that creepage throughout this manuscript refers only to the longitudinal creepage. The lateral and spin creepages are considered negligible due to the controlled conditions of the conducted experiments.

\section{Multiple regression models}

To develop a multiple regression model that involves the variables mentioned earlier, one needs to first study the main effect of each on traction, independent of the others, and find the functional form that best describes the relationship between them. The extracted features are then combined into a multiple regression model, and pairwise interactions between the variables are examined via model selection methods. The corresponding single regression models were introduced in an earlier study by the authors [19]. They are also covered in detail in [18]. This 
section extends the analysis from single- to multiple-regression. The multiple regression models developed here not only account for the effects of wheel load, percent-creepage, and AoA, but also the interaction among them. The analyses in this section are conducted within the classical linear regression framework, using the training data sets. The statistical programming and computations throughout this study are done using $\mathrm{R}$, an open-source programming language that is commonly used for statistical computing along with its supporting libraries [21].

There are a couple of (implicit) guidelines that must be considered in extending single-regression models to a multiple-regression model:

1. There is no multicollinearity among the independent variables

2. The sign of the main effects in the multiple regression model must match the corresponding single regression model

The latter condition makes sure the main effect of each independent variable acts in the direction of its true influence on the response, while the former ensures that none of the independent variables is a linear combination of the other variables. Table 2 includes the correlation matrix for the independent variables in the data set. It shows that there is no strong pairwise correlation between the explanatory variables, therefore there are no issues in this regard. One must note that the key term here is linear. In other words, the correlation matrix does not reveal anything about the non-linear relationship between any two columns. The match or mismatch of the sign of the main effects in the multiple regression model may be checked during the model selection process. 
Table 2: Pairwise correlation of explanatory variables

\begin{tabular}{cccc}
\hline & Load & AoA & Creepage \\
\hline Load & 1.00 & -0.057 & 0.002 \\
AoA & -0.057 & 1.00 & 0.00 \\
Creepage & 0.002 & 0.00 & 1.00 \\
\hline
\end{tabular}

\section{Multiple regression model for longitudinal force}

In [19], it was concluded that the longitudinal force has a linear relationship with the wheel load and quadratic relationships with the creepage and AoA. Here, we attempt to develop a multiple regression model for the longitudinal force based on the single models presented in [19]. Linear regression analysis could be used to capture non-linear relationships like the one between creepage and longitudinal force. The statistical aspects of this point are rigorously evaluated and discussed in another publication by the authors [19].

\subsection{Superposition of main effects}

We start with the simplest case, the superposition of the main effects of independent variables on the longitudinal force. The regression summary for the model is shown in Table 3:

$$
\begin{gathered}
\text { Longitudinal Force }_{i}=\beta_{0}+\beta_{1} \text { Load }_{i}+\beta_{2} \text { Creepage }_{i}+\beta_{3} \text { AoA }^{2}{ }_{i}+\beta_{4} \text { Creepage }_{i}{ }_{i}+\varepsilon_{i} \\
\text { where } \varepsilon_{i} \sim \mathcal{N}\left(0, \mathbb{I}_{4} \sigma^{2}\right)
\end{gathered}
$$

The model explains $85 \%$ of the variability in the data, the signs match with the single models, and all the coefficient estimates are statistically significant at less than $1 \%$ confidence level.

Table 3: Regression summary for regressing longitudinal force on the main effects of explanatory variables 


\begin{tabular}{lccc}
\hline \multicolumn{3}{c}{ Longitudinal Force } \\
Predictors & Estimates & $C I$ & $p$ \\
\hline (Intercept) & -1251.67 & $-1254.65--1248.69$ & $<\mathbf{0 . 0 0 1}$ \\
Load & 0.33 & $0.33-0.33$ & $<\mathbf{0 . 0 0 1}$ \\
AoA $^{2}$ & -712.63 & $-715.62--709.64$ & $<\mathbf{0 . 0 0 1}$ \\
Creepage & 2034.47 & $2028.49-2040.45$ & $<\mathbf{0 . 0 0 1}$ \\
Creepage $^{2}$ & -511.07 & $-513.98--508.17$ & $<\mathbf{0 . 0 0 1}$ \\
& & & \\
Observations & 1344000 & & \\
$\mathrm{R}^{2} / \mathrm{R}^{2}$ adjusted & $0.852 / 0.852$ & \\
\hline
\end{tabular}

\subsection{Stepwise model selection via Bayes Information Criterion (BIC)}

The model selected by the step function with Bayes Information Criterion (BIC) as the selection criteria is presented in Table 4. It incorporates all the possible pairwise interactions of independent variables and explains $95 \%$ of the variability in the data. This means that the combined effect of the six interaction terms accounts for only $10 \%$ of the variability in the data, approximately $1.6 \%$ for new each term added to the initial model. Even though there is no mismatch in terms of the signs of independent variables in the single models and the model explains much of the variability in the data, this model is the most complicated as far as the pairwise interactions are concerned and there probably exists some simpler model that performs as well.

Table 4: Regression summary for the result of stepwise model selection via BIC for longitudinal force

\begin{tabular}{lccc}
\hline & \multicolumn{3}{c}{ Longitudinal Force } \\
Predictors & Estimates & $C I$ & $p$ \\
\hline (Intercept) & 54.18 & $49.10-59.26$ & $<\mathbf{0 . 0 0 1}$
\end{tabular}




\begin{tabular}{|c|c|c|c|}
\hline Load & 0.09 & $0.09-0.09$ & $<0.001$ \\
\hline Creepage & 184.16 & $173.01-195.30$ & $<0.001$ \\
\hline $\mathrm{AoA}^{2}$ & -944.81 & $-961.92--927.69$ & $<0.001$ \\
\hline Creepage $^{2}$ & -423.38 & $-433.56--413.20$ & $<0.001$ \\
\hline Load * Creepage & 0.43 & $0.43-0.43$ & $<0.001$ \\
\hline Load $*$ AoA $^{2}$ & -0.16 & $-0.16--0.16$ & $<0.001$ \\
\hline Load $*$ Creepage $^{2}$ & -0.11 & $-0.11--0.11$ & $<0.001$ \\
\hline Creepage $*$ AoA $^{2}$ & 773.42 & $747.19-799.65$ & $<0.001$ \\
\hline Creepage $*$ Creepage $^{2}$ & 129.16 & $125.81-132.52$ & $<0.001$ \\
\hline AoA $^{2} *$ Creepage $^{2}$ & -67.42 & $-77.11--57.72$ & $<0.001$ \\
\hline Observations & \multicolumn{3}{|l|}{1344000} \\
\hline $\mathrm{R}^{2} / \mathrm{R}^{2}$ adjusted & \multicolumn{3}{|c|}{$0.952 / 0.952$} \\
\hline
\end{tabular}

The intuition behind interaction terms, such as the ones in Table 4, is that the relationship between the independent variable, in our case longitudinal force, and the dependent variables is beyond the simple superposition of their individual effects. For instance, the statistically significant coefficient for Load $*$ Creepage in the above multiple regression model indicates that the simultaneous changes in the wheel load and creepage have a measurable effect on longitudinal force, in addition to the individual effects of wheel load and creepage.

\subsection{Least Absolute Shrinkage and Selection Operator (LASSO) \& best subset selection}

Taking a reverse approach to the previous section enables us to eliminate terms from the model.

Table 5 shows the coefficients estimated by the Least Absolute Shrinkage and Selection Operator (LASSO), which is a penalized regression model, for the model including the main 
effects and all possible interaction terms except for Creepage $*$ Creepage ${ }^{2}$.

Table 5: LASSO coefficient estimates for the longitudinal force model including all interactions

Variable Load Creepage AoA ${ }^{2}$ Creepage ${ }^{2}$ Load $^{*}$ Creepage Load*AoA ${ }^{2}$ Load $^{*}$ Creeoage $^{2}$ Creepage $^{*}$ AoA $^{2}$ Creepage $^{2 *}$ AoA $^{2}$

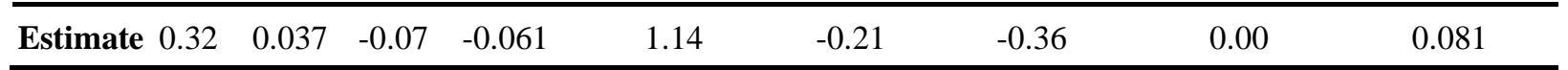

The signs match with the single models and the effect of Creepage $* A o A^{2}$ was estimated to be zero by the penalized regression model. We combine this result with that of the Best Subset Selection method to test if the combined results could help us select a simpler and more descriptive model.

The Best Subset Selection method is analogous to the stepwise selection approach except that the numbers of selection criteria are different. Figure 3 shows the progression of various selection criteria during the best subset selection process. 

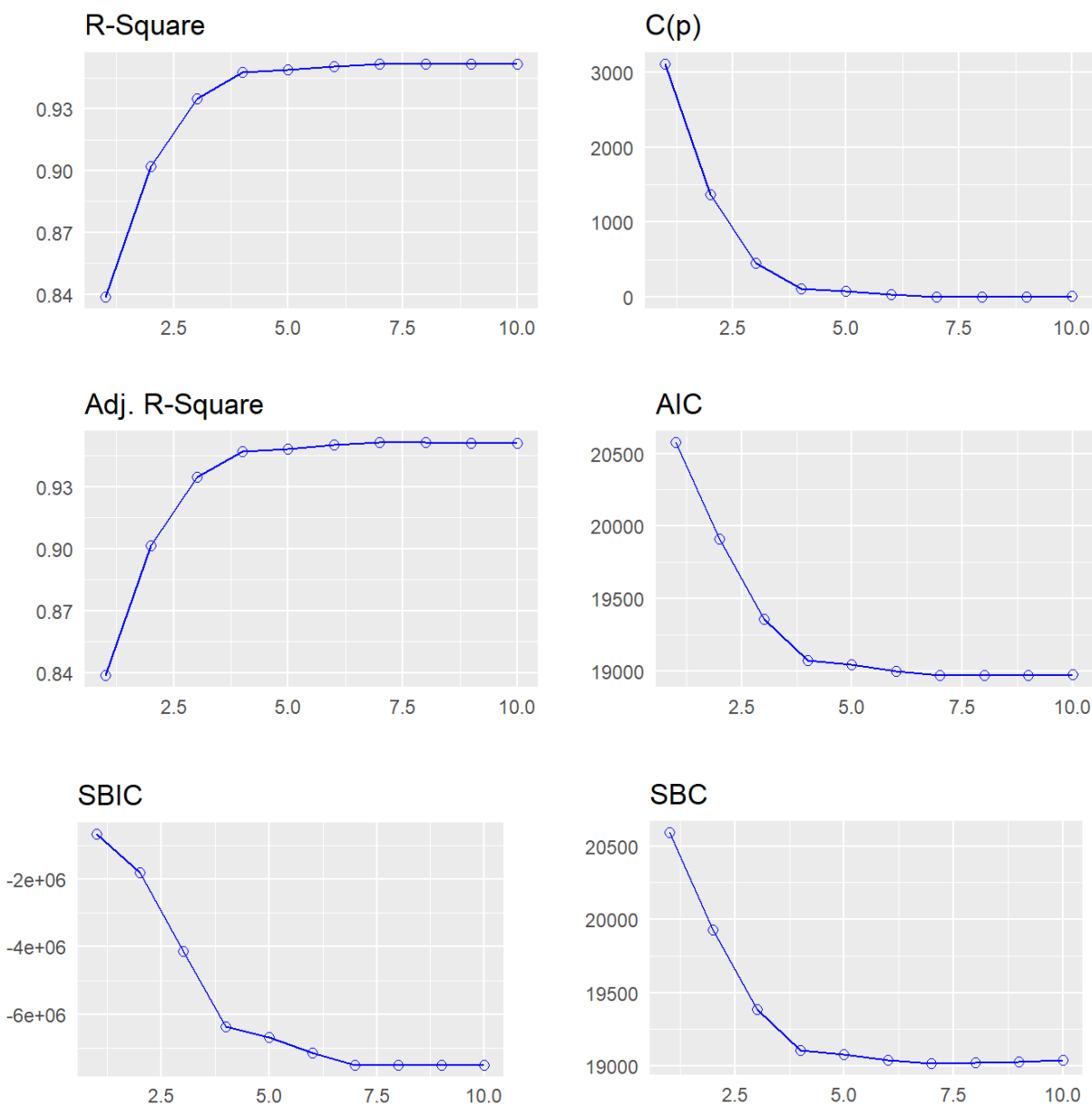

Figure 3: Best subset selection criteria for choosing among the longitudinal force models

The curve for $\mathrm{R}^{2}$ and AIC (Akaike Information Criterion) after the fourth step reaches a plateau. The same is observed for the other measures displayed in the figure. Each point on the curves corresponds to a model during the selection process as the step function adds new terms to the initial model.

The first four models (represented by the first four points across the subplots) are:

$$
\begin{aligned}
& \text { Longitudinal Force }_{i}=\beta_{0}+\beta_{1} \text { Load }_{i} * \text { Creepage }_{i}+\varepsilon_{i} \\
& \text { Longitudinal Force }_{i}=\beta_{0}+\beta_{1} \text { Load }_{i} * \text { Creepage }_{i}+\beta_{2} \operatorname{Load}_{i} * \operatorname{Creepage}_{i}+\varepsilon_{i} \\
& \text { Longitudinal Force }_{i}=\beta_{0}+\beta_{1} \text { Load }_{i}+\beta_{2} \operatorname{Load}_{\boldsymbol{i}} * \operatorname{Creepage}_{\boldsymbol{i}}+\beta_{3} \operatorname{Load}_{\boldsymbol{i}} * \boldsymbol{A o A}_{\boldsymbol{i}}{ }_{i}+\varepsilon_{i}
\end{aligned}
$$




\section{Longitudinal Force F $_{i}$}

$$
\begin{aligned}
& =\beta_{0}+\beta_{1} \operatorname{Load}_{i}+\beta_{2} \operatorname{Load}_{i} * \text { Creepage }_{i}+\beta_{3} \operatorname{Load}_{i} * \operatorname{AoA}_{i}{ }_{i} \\
& +\beta_{4} \operatorname{Load}_{i} * \text { Creepage }_{i}{ }_{i}+\varepsilon_{i}
\end{aligned}
$$

The selection process starts with the interaction of the wheel load and creepage in the first model, and then the interactions of wheel load with Creepage ${ }^{2}$ and $A o A^{2}$ are added in the second and third models. Finally, in the fourth model, the main effect of the wheel load is added to the three interaction terms. This is congruent with the earlier results from the LASSO. We saw that the wheel load and creepage were the most important variables with the wheel load far ahead of creepage. This translated into the first model and the fourth model exhibiting the prevalence of the effect of the wheel load by incorporating the main effect and all the pairwise interactions of the wheel load. This makes the fourth model in the subset selection process one of the strongest candidates for modeling the longitudinal force.

\subsection{Selected model}

In summary, we are left with the main effects of the explanatory variables plus four potential interaction terms (Load $* A o A^{2}$, Load $*$ Creepage, Load $*$ Creepage $^{2}, A o A^{2} *$ Creepage $^{2}$ ) with a maximum effect of explaining 10 percent of the variability in the data. Different combinations of interaction terms and the main effects were tried out to pick the simplest model that explains the data as much as possible. Many of these models had one of the following issues:

a) The sign of main effects match with that of the single regression model

b) The coefficient estimates were not significant at a $10 \%$ or higher confidence level

c) The potential model explained variability in the data no more than the superposition of main effects $\left(\mathrm{R}^{2}<90 \%\right)$ 
These results in tandem with the developed single regression models suggest that the fourth model of the best subset selection is the simplest and most interpretable model for the longitudinal force. Table 6 presents the regression summary for the selected model.

$$
\begin{aligned}
& \text { Longitudinal Force }_{i}=\beta_{0}+\beta_{1} \operatorname{Load}_{i}+\beta_{2} \text { Load }_{i} * \text { Creepage }_{i}+\beta_{3} \operatorname{Load}_{i} * \text { AoA }_{i}{ }_{i}+ \\
& \beta_{4} \operatorname{Load}_{i} * \text { Creepage }_{i}{ }_{i}+\varepsilon_{i} \quad \text { where } \varepsilon_{i} \sim \mathcal{N}\left(0, \mathbb{I}_{4} \sigma^{2}\right)
\end{aligned}
$$

Table 6: Regression summary for the selected model for longitudinal force

\begin{tabular}{lccc}
\hline & \multicolumn{3}{c}{ Longitudinal Force } \\
Predictors & Estimates & $C I$ & $p$ \\
\hline (Intercept) & -132.92 & $-160.61--105.23$ & $<\mathbf{0 . 0 0 1}$ \\
Load & 0.11 & $0.10-0.12$ & $<\mathbf{0 . 0 0 1}$ \\
Load*Creepage & 0.38 & $0.36-0.40$ & $<\mathbf{0 . 0 0 1}$ \\
Load*AoA $^{2}$ & -0.14 & $-0.15--0.14$ & $<\mathbf{0 . 0 0 1}$ \\
Load*Creepage $^{2}$ & -0.08 & $-0.09--0.07$ & $<\mathbf{0 . 0 0 1}$ \\
& & & \\
Observations & 1344 & & \\
$\mathrm{R}^{2} / \mathrm{R}^{2}$ adjusted & $0.947 / 0.947$ & \\
\hline
\end{tabular}

\section{Multiple regression model for lateral force}

A similar analysis was carried out to develop a multiple regression model for lateral traction.

$$
\begin{gathered}
\text { Lateral Force }_{i}=\beta_{0}+\beta_{1} \text { Load }_{i}+\beta_{1} \text { AoA }_{i}+\beta_{3} \operatorname{Load}_{i} * \text { AoA }_{i}+\beta_{4} \text { AoA }_{i} * \text { Creepage }_{i}+\varepsilon_{i} \\
\text { where } \varepsilon_{i} \sim \mathcal{N}\left(0, \mathbb{I}_{4} \sigma^{2}\right)
\end{gathered}
$$

Table 7 shows the regression summary for the selected model. More details regarding single regression models and model selection process are included in [18]. The wheel load influencing the lateral force through the taper of the wheel shows itself with a very small but statistically significant coefficient estimate for wheel load. Note that the selected multiple 
regression model suggests that the relationship among the variables is beyond the superposition of main effects, similar to longitudinal traction.

Table 7: Regression summary for the selected model for lateral force

\begin{tabular}{lccc}
\hline \multicolumn{3}{c}{ Lateral Force } \\
Predictors & Estimates & $C I$ & $p$ \\
\hline (Intercept) & -74.23 & $-109.07--39.40$ & $<\mathbf{0 . 0 0 1}$ \\
Load & 0.14 & $0.14-0.15$ & $<\mathbf{0 . 0 0 1}$ \\
AoA & 1209.21 & $1120.27-1298.15$ & $<\mathbf{0 . 0 0 1}$ \\
AoA*Load & 0.46 & $0.45-0.47$ & $<\mathbf{0 . 0 0 1}$ \\
AoA*Creepage & -986.28 & $-1044.12--928.44$ & $<\mathbf{0 . 0 0 1}$ \\
Observations & 1344 & & \\
$\mathrm{R}^{2} / \mathrm{R}^{2}$ adjusted & $0.952 / 0.952$ & \\
\hline
\end{tabular}

\section{Comparison with CONTACT}

The regression model predictions ("out-of-sample" results) for lateral and longitudinal tractions are compared with CONTACT, an established wheel-rail contact dynamics software. The input parameters used to run simulations in CONTACT are identical to those used for making predictions by the multiple regression model to allow for a direct comparison. Other input parameters for CONTACT simulation are set based on the roller rig's specifications as listed in Table 8.

Table 8: Input parameters for CONTACT simulation

\begin{tabular}{ll}
\hline CONTACT Parameter & Set Value \\
\hline Static coefficient of friction & 0.5 \\
Kinetic coefficient of friction & 0.5 \\
Poisson ratio of the roller and the wheel & 0.28
\end{tabular}


Modulus of rigidity for the roller and the wheel

Layer thickness

Shear elastic modulus of interface layer

Initial yield limit at which plasticity starts to occur

Rate of increase of the yield limit with accumulated plastic deformation
$82000\left(\mathrm{~N} / \mathrm{mm}^{2}\right)$

$0.002(\mathrm{~mm})$

$500\left(\mathrm{~N} / \mathrm{mm}^{2}\right)$

$480\left(\mathrm{~N} / \mathrm{mm}^{2}\right)$

$12000\left(\mathrm{~N} / \mathrm{mm}^{3}\right)$

Figure 4 shows the actual longitudinal and lateral measurements from the experiment ("testing set"), regression model predictions, and CONTACT results. Note that each data point on the $\mathrm{x}$-axis in Figure 4 represents an input parameter set i.e., a combination of percentcreepage, AoA, and wheel load that was initially used to conduct the experiment. In other words, the input parameters used to obtain the results in Figure 4 represent $30 \%$ of the entire data set which were randomly selected and set aside for the sole purpose of evaluating the accuracy of the regression and CONTACT models. As noted earlier, the training of the multiple regression models is done using the training set and outside of the testing set to avoid data bias.

As shown for longitudinal traction in Figure 4, three curves are in excellent agreement. The Root-Mean-Squared Error (RMSE) of the predictions by the regression model and CONTACT with respect to actual measurements is $294 \mathrm{~N}$ and $366 \mathrm{~N}$, respectively. For a range of $6,666 \mathrm{~N}$ in longitudinal traction measurements, the RMSE amounts to $4.4 \%$ and 5.5\%, respectively. 

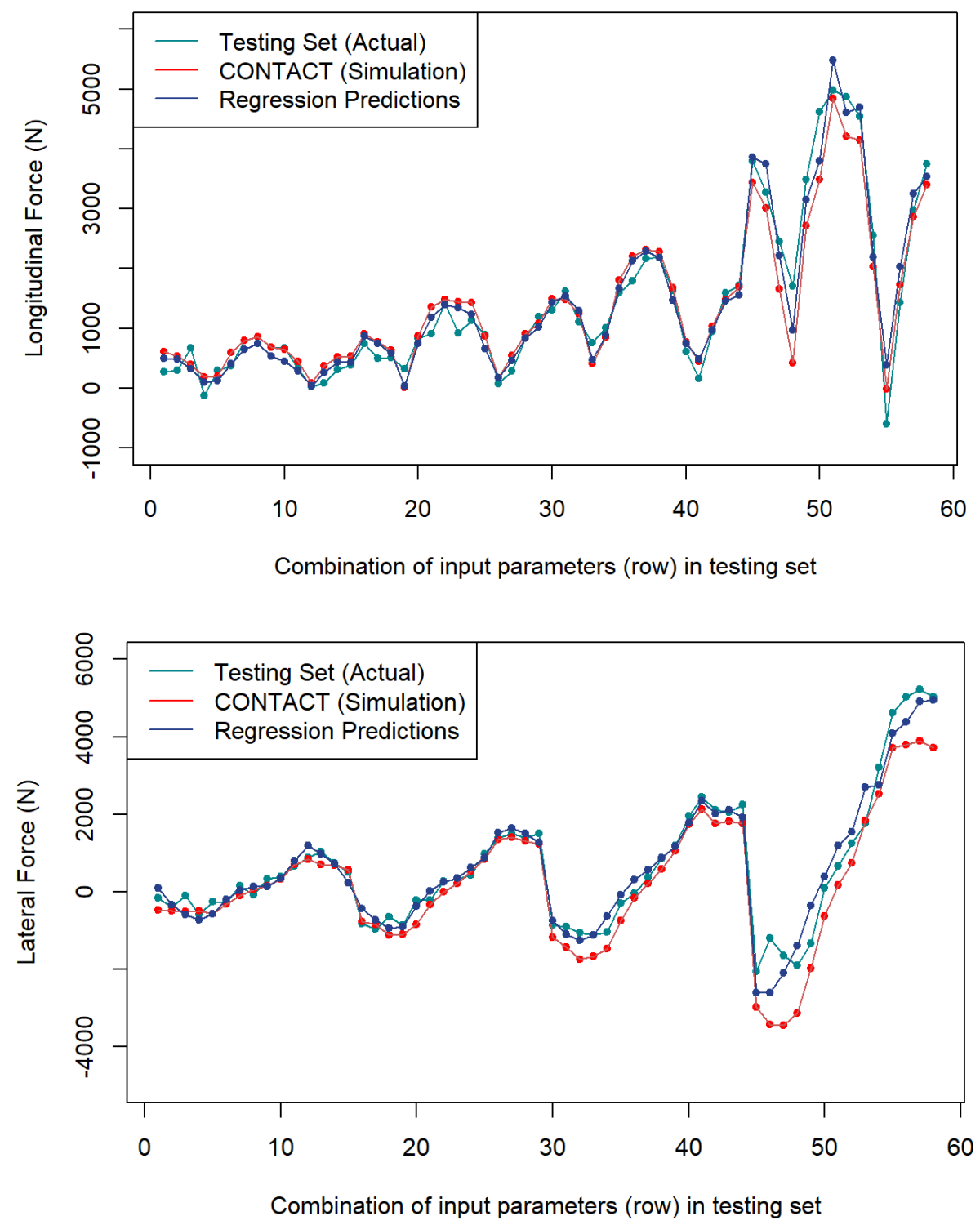

Figure 4: Actual experiment measurements (testing set), CONTACT simulation

The results indicate that both models provide a good agreement with the actual measurements, except that the regression model provides an algebraic expression that can be easily solved for parameters outside of the predefined experiment conditions, whereas such an extrapolation cannot be done by CONTACT without a new simulation run. The results for the 
lateral traction as shown in the lower panel of Figure 4, are as favorable as the longitudinal traction. The RMSE is $4.5 \%$ and $7.5 \%$ for the regression model and CONTACT, respectively. The larger RMSE for CONTACT is attributed to wheel and rail (in our case, roller) conditions that may not be easily measured with sufficient accuracy.

Although for this study we carefully measured various parameters that are needed for CONTACT simulation, any inaccuracy in measurements or parameters that cannot be easily measured contributes to lower accuracies that are present in the CONTACT predictions.

\section{Conclusion}

The results of past studies on evaluating the individual effects of wheel load, percent-creepage, and Angle of Attack (AoA) through single-regression models are extended to multiple regression models in which the combined effect of the parameters on longitudinal and lateral traction are studied. The multiple regression models are offered as an alternative to well-known wheel-rail contact modeling tools such as CONTACT. Certainly, such accurate regression models would require access to a large volume of data, such as what is used in this study, but the ease of instrumentation and data collection in recent years has made clearing this hurdle far easier.

The multiple regression results indicate that the relationship among the variables is well beyond a simple superposition of each. They prove that not all of the features introduced in single-regression models are significantly influential for longitudinal and lateral traction when the combined effects of the variables are evaluated. The multiple regression model for longitudinal traction pivots on the wheel load and its interaction with the other variables such as percent-creepage and AoA. This makes intuitive sense as it accurately reflects the significant 
influence of wheel load on traction. On the other hand, the multiple regression model for lateral traction indicates that the AoA and its interactions with wheel load and percent-creepage are the most essential elements in accurately predicting lateral forces.

This study bridges the gap between the well-established models for the wheel-rail contact dynamics and the state-of-the-art machine/deep learning methods that are used for parameter estimation and predictions by statistically modeling the contact dynamics using experimental data. The contribution from this research becomes more obvious if we take a closer look at the opposite ends of the spectrum. On one end, we have longitudinal and lateral traction models that take a purely Hertzian approach, which starts from theory and the assumptions that it entails and finds its way to practice via numerical algorithms and software. On the other end of the spectrum, we have deep neural networks and non-parametric methods that start from experimental data, are not concerned with the mathematical functional relationships between the independent and dependent variable in any way, and mostly focus on out-of-sample performance and the accuracy of predictions in practice. The developed statistical models borrow the strengths of both approaches by letting the data speak for itself. They employ mathematical functions to define the relationship between independent and dependant variables but make conclusions based on the performance of the model on unseen data. Unlike the two approaches, they are capable of providing a margin for the certainty of predictions in addition to the point estimates. In other words, other approaches can predict traction forces for a specific group of input variables but there is no indicator or measure to quantify how close these point estimates or predictions are to laboratory or field data. The statistical models bridge this gap by capturing the relationship between the dependent and independent variables in such a way that could be 
extrapolated to un-tested scenarios, while also providing a range for the accuracy of their predictions."

\section{References}

[1] Meymand SZ, Keylin A, Ahmadian M. A survey of wheel-rail contact models for rail vehicles. Veh Syst Dyn. 2016;54:386-428. Available from: https://doi.org/10.1080/00423114.2015.1137956.

[2] Baek KS, Kyogoku K, Nakahara T. An experimental investigation of transient traction characteristics in rolling-sliding wheel/rail contacts under dry-wet conditions. Wear. 2007;263:169-179.

[3] Spiryagin M, Polach O, Cole C. Creep force modelling for rail traction vehicles based on the Fastsim algorithm. Veh Syst Dyn. 2013;51:1765-1783.

[4] Santamaria J, Vadillo EG, Gomez J. Influence of creep forces on the risk of derailment of railway vehicles. Veh Syst Dyn. 2009;47:721-752.

[5] Jin X, Wu P, Wen Z. Effects of structure elastic deformations of wheelset and track on creep forces of wheel/rail in rolling contact. Wear. 2002;253:247-256.

[6] Guan Q, Zeng J, Jin X. An angle of attack-based derailment criterion for wheel flange climbing. Proc Inst Mech Eng Part F J Rail Rapid Transit. 2014;228:719-729.

[7] Marquis B, Greif R. Application of nadal limit in the prediction of wheel climb derailment. 2011 Jt Rail Conf JRC 2011. 2011;273-280.

[8] O’Shea JJ, Shabana AA. Analytical and numerical investigation of wheel climb at large angle of attack. Nonlinear Dyn. 2016;83:555-577.

[9] Alonso A, Guiral A, Baeza L, et al. Wheel-rail contact: Experimental study of the creep forces-creepage relationships. Veh Syst Dyn. 2014;52:469-487.

[10] Hosseinipour M. Electromechanical Design and Development of the Virginia Tech Roller Rig Testing Facility for Wheel-Rail Contact Mechanics and Dynamics. Phd Diss. 2016. 
[11] Nachtigall K, Voget S. A genetic algorithm approach to periodic railway synchronization. Comput Oper Res. 1996;23:453-463.

[12] Parkinson H, Iwnicki S. An Intelligent Track Monitoring System. Available from: http://www.railtechnologyunit.com.

[13] Persson I, Iwnicki SD. Optimisation of railway wheel profiles using a genetic algorithm. Veh Syst Dyn. 2004;41:517-526.

[14] Shebani A, Iwnicki S. Prediction of wheel and rail wear under different contact conditions using artificial neural networks. Wear. 2018;406-407:173-184. Available from: https://doi.org/10.1016/j.wear.2018.01.007.

[15] Keylin A. Analytical Evaluation of the Accuracy of Roller Rig Data for Studying Creepage in Rail Vehicles. Virginia Polytechnic Institute and State University; 2012. Available from:

https://vtechworks.lib.vt.edu/bitstream/handle/10919/49607/Keylin_A_T_2013.pdf?seque nce $=1$.

[16] Radmehr A, Tajaddini A, Marquis B, et al. Virginia Tech-Federal Railroad Administration Roller Rig Measurement Capabilities And Baseline Measurements. Proc 2019 ASME Jt Rail Conf JRC2019.

[17] Radmehr A, Ahangarnejad AH, Pan Y, et al. Wheel-rail contact patch geometry measurement and shape analysis under various loading conditions. 2020 Jt Rail Conf JRC 2020. St. Louis, MO, USA; 2020.

[18] Hosseini S. A Statistical Approach To Modeling Wheel-Rail Contact Dynamics. Master's Thesis, Virginia Polytechnic Institute and State University; 2020. Available from: https://vtechworks.lib.vt.edu/handle/10919/101864.

[19] Hosseini S, Hosseinian Ahangarnejad A, Radmehr A, et al. A Statistical Approach To Evaluating Wheel-Rail Contact Dynamics. Proc 2021 ASME Jt Rail Conf JRC2021. April 20-21, 2021, Virtual, Online: Proceedings of the 2021 Joint Rail Conference JRC2021;

[20] Radmehr A, Kothari K, Ahmadian M. Evaluating The Effect Of Natural Third Body 
Layers On Friction Using The Virginia Tech Roller Rig. Proc 2019 ASME Jt Rail Conf JRC2019 April 10-12, 2019, Snowbird, UT, USA. 2019;

[21] R-Core-Team. R: A language and environment for statistical computing. R Foundation for Statistical Computing, Vienna, Austria; 2020. Available from: https://www.r-project.org/. 